\begin{tabular}{|c|l|}
\hline Title & Resonance and mode conversion of phonons scattered by superlattices with inhomogeneity \\
\hline Author(s) & Mizuno, Seiji \\
\hline Citation & $\begin{array}{l}\text { Physical Review B, 68, 193305 } \\
\text { https://doi.org/10.1103/PhysRevB.68.193305 }\end{array}$ \\
\hline Issue Date & 2003-11-18 \\
\hline Doc URL & http://hdl.handle.net/2115/5704 \\
\hline Rights & Copyright $\odot 2003$ A merican Physical Society \\
\hline Type & article \\
\hline File Information & PRB68.pdf \\
\hline
\end{tabular}

Instructions for use 


\title{
Resonance and mode conversion of phonons scattered by superlattices with inhomogeneity
}

\author{
Seiji Mizuno \\ Department of Applied Physics, Hokkaido University, Sapporo 060-8628, Japan \\ (Received 2 April 2003; revised manuscript received 18 August 2003; published 18 November 2003)
}

\begin{abstract}
For phonons propagating through superlattices, "intermode" Bragg reflections due to the coupling of different polarization modes can occur besides ordinary "intramode" Bragg reflections, and corresponding frequency gaps are classified according to the types of Bragg reflection. We demonstrate the difference between localized modes generated in different types of frequency gaps in superlattices with inhomogeneity. In particular, we show for a superlattice with a free surface, a "perfect" resonant reflection associated with surfacelocalized modes is realized inside frequency gaps in which intermode Bragg reflections occur. This resonance can be interpreted as resonant tunneling through a symmetric double-barrier potential.
\end{abstract}

DOI: 10.1103/PhysRevB.68.193305

PACS number(s): 68.65.-k, 62.65.+k, 63.20.-e

The noticeable behavior of phonons in periodic superlattices (SL's) is mainly related to the appearance of frequency gaps due to Bragg reflections of phonons. ${ }^{1,2}$ Since Bragg reflections occur essentially as a result of the interference of waves, they are also seen in the propagation of photons and electrons through periodic media. ${ }^{3}$ In the case of phonons, there exists an inherent type of Bragg reflection in addition to ordinary Bragg reflections. ${ }^{4}$ When phonons are injected into interfaces of a SL at an angle, three modes of propagation-one longitudinal (L) and two transverse (T) are involved in the reflection and transmission processes. This leads to coupling between the modes and mode conversion at the interface. As a result of the multiple reflection by the interfaces of the periodic SL, "intermode" Bragg reflections can occur besides ordinary "intramode" Bragg reflections. For example, incident L phonons can be Bragg reflected as $\mathrm{T}$ phonons, i.e., the amplitudes of the reflected $\mathrm{T}$ phonons add up in phase but those of the reflected $\mathrm{L}$ phonons are canceled out. Therefore, the corresponding frequency gaps should be classified when mode-dependent characteristics of phonon propagation are investigated.

On the other hand, a lot of studies have shown that an inhomogeneity embedded in a periodic SL causes localized vibrational modes inside the frequency gaps. ${ }^{5-14}$ It is expected that these localized modes also have different features according to the types of frequency gaps in which the localized modes exist. However, this has not been well recognized till date. In the present paper, we study theoretically the localized modes generated in the different types of frequency gaps. We examine the resonant interaction of localized modes with acoustic phonons injected into SL's with inhomogeneity at an angle. We calculate the reflectance of phonons scattered by SL's with a free surface or defect layer. In particular, we show for the SL with a free surface that a "perfect" resonant reflection is realized inside the frequency gaps in which intermode Bragg reflections occur.

First of all, we briefly summarize the features of phonons propagating through a periodic SL without inhomogeneity and emphasize the characteristics of the frequency gap in which intermode Bragg reflections occur. As a numerical example, we show in Fig. 1(a) the calculated dispersion relation of acoustic phonons propagating through a (001) GaAs/ AlAs SL at an angle of $25^{\circ}$ (in GaAs layers). The calculation is carried out by means of the transfer matrix method. In the present calculation, we adopt the isotropic continuum approximation for each constituent layer of the SL. Under this condition, the phonon modes polarized in the sagittal plane are decoupled from the horizontally polarized shear (SH) mode. We consider the coupled $\mathrm{L}$ and $\mathrm{T}$ vibrations in the sagittal plane. Frequency gaps are generated inside the miniBrillouin zone as well as at the zone center and boundary. The condition for ordinary intramode Bragg reflections can be written as

$$
2 q=m G_{0}
$$

with the order of the reflection denoted as $m$. Here, $q$ $=2 \pi \cos \theta / \lambda$ is the component of the wave vector normal to the interfaces, $\theta$ is the angle the wave vector makes with the interface normal, $\lambda$ is the wavelength of the phonons, and $G_{0}=2 \pi / D$ is the reciprocal vector of the SL with periodicity $D$. The condition (1) is satisfied at the center and edges of the mini-BZ for both $\mathrm{L}$ and $\mathrm{T}$ modes. The corresponding gaps are labeled with LL and TT in Fig. 1(a). On the other hand, the condition for intermode Bragg reflections was given by ${ }^{4}$

$$
q_{L}+q_{T}=m G_{0},
$$

where $q_{L}$ and $q_{T}$ are the normal components of the wave vectors of the $\mathrm{L}$ and $\mathrm{T}$ phonons, respectively. This condition (2) is satisfied at the points where the folded dispersion curve of the $\mathrm{T}$ phonons intersect with that of the $\mathrm{L}$ phonons. The corresponding frequency gap is labeled with LT in Fig. 1(a). To demonstrate the qualitative difference among the LL, TT, and LT gaps, the calculated transmittance and reflectance are shown in Fig. 1(b). The incident phonons are assumed to be $\mathrm{L}$ phonons in this numerical example. The solid (broken) line is for the transmitted L (T) phonons. From Fig. 1(b), we can see a variety of propagation patterns in each frequency gap. Within the LL and LT gaps, there is no transmitted phonon. Though the incident $\mathrm{L}$ phonons are Bragg reflected as $\mathrm{L}$ phonons within the LL gap in which Eq. (1) is satisfied, they are reflected with mode conversion within the LT gap in which Eq. (2) is satisfied. Within the TT gap, the incident L phonons are transmitted as L phonons. That is, Bragg reflections do not occur because the TT gap is not a real frequency 
(a) Dispersion

(b) Transmittance and Reflectance (incident phonon: L)

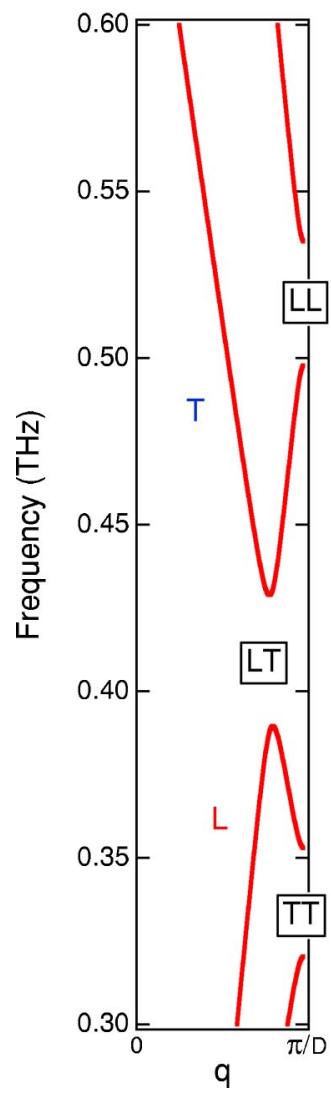

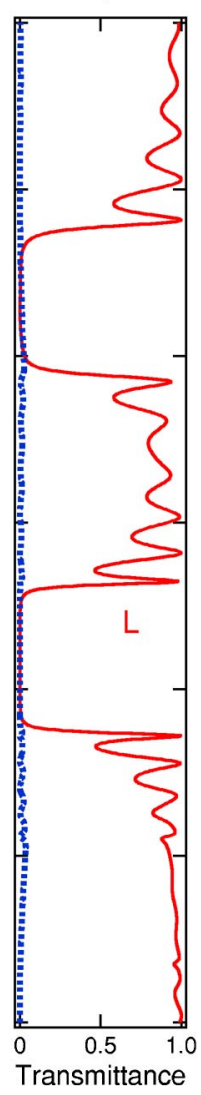

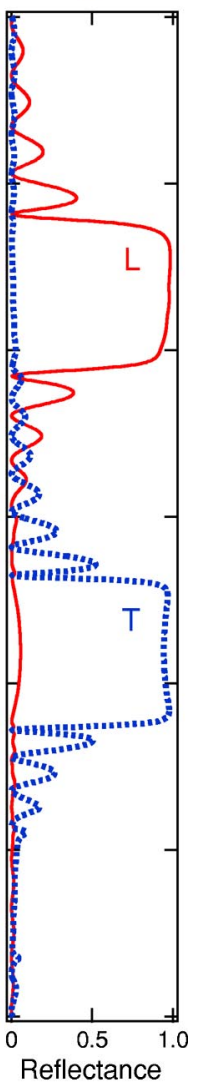

FIG. 1. (a) Dispersion relation of phonons propagating at oblique incidence in a (001) GaAs/AlAs SL, with $\theta_{L}=25$ in GaAs. The unit period of the SL consists of $(\mathrm{GaAs})_{15}(\mathrm{AlAs})_{15}$. (b) The transmittance and reflectance of phonons incident on a (001) GaAs/ AlAs SL with 25 periods. The substrate and detector layer are assumed to be GaAs. The incident phonons are assumed to be $\mathrm{L}$ phonons. The solid (broken) lines are for the transmitted L (T) phonons.

gap for $\mathrm{L}$ phonons. Within this frequency range, in fact, there exists the dispersion curve corresponding to the L mode [see Fig. 1(a)].

Here, we note that the behavior in each gap is essentially independent of the incident angle. Main difference concerns the width of the LL, TT, and LT gaps. For a small angle, the coupling of the $\mathrm{L}$ and $\mathrm{T}$ modes hardly occur. As a result, the width of the LT gap is narrow and that of the LL (or TT) gap is wide. For a larger angle, the width of the LT gap is wider but that of the LL (TT) gap becomes narrower. When $\theta_{L}$ $=25$, the LL, TT, and LT gaps have similar widths in the present numerical example. To demonstrate the difference in the LL, TT, and LT gaps within one figure, we chose $\theta_{L}$ $=25$.

Next, we examine localized modes generated inside each gap. As a first example, we consider the vibrational modes localized at an impurity layer embedded in a periodic SL. Figure 2 shows the transmittance and reflectance calculated for the GaAs/AlAs SL with a GaAs impurity layer. Here, we note that the present system is symmetric. That is, the GaAs layer is sandwiched between the same two GaAs/AlAs SL,

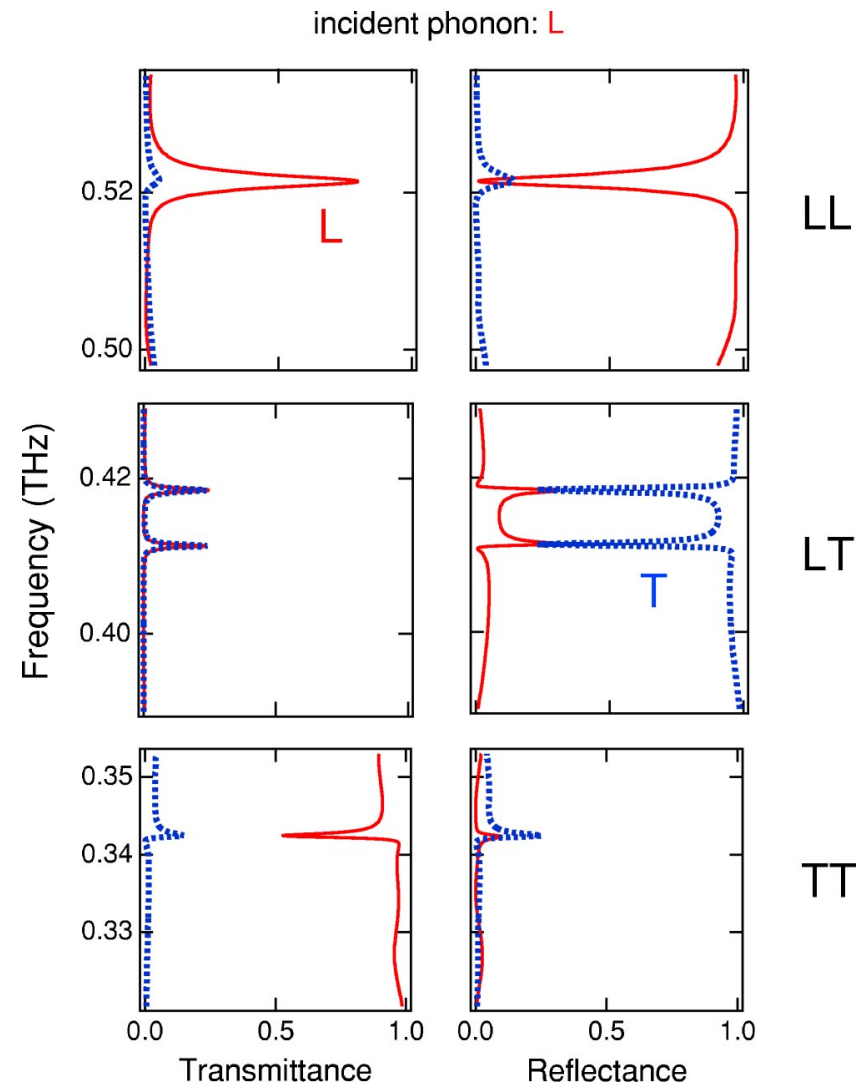

FIG. 2. The transmittance and reflectance of phonons obliquely incident on a (001) GaAs/AlAs SL with a GaAs impurity layer. The substrate and detector layer are also assumed to be GaAs. The incident phonons are assumed to be L phonons. The solid (broken) line is for the transmitted $\mathrm{L}(\mathrm{T})$ phonons.

and the substrate and detector layer are also assumed to be GaAs. Inside the LT gap, we can see the resonant peaks in transmittance and reflectance for both $\mathrm{L}$ and $\mathrm{T}$ modes. These peaks are due to the localized vibrational modes. This means that additive interference between the incident $\mathrm{L}$ phonons and reflected $\mathrm{T}$ phonons is violated at the eigenfrequency of the localized mode. In the LT gap, the localized modes consist of both $\mathrm{L}$ and $\mathrm{T}$ components with imaginary wave numbers (that is, this gap is a real frequency gap for the coupled $\mathrm{L}$ and $\mathrm{T}$ phonons). As a result of the resonant interaction with the localized mode, the incident L phonons are trapped in the impurity layer for a while and then transmitted and reflected as $\mathrm{L}$ and $\mathrm{T}$ phonons. That is, after the scattering process the incident $\mathrm{L}$ phonons are equally divided into four pieces. In the LL gap, the intramode Bragg reflection is broken off at the eigenfrequency of the localized mode. That is, additive interference between the incident $\mathrm{L}$ phonons and reflected $\mathrm{L}$ phonons is violated. As a result of the resonant interaction, most of the phonons are transmitted as L phonons. The rest are transmitted and reflected as the $\mathrm{T}$ phonons since only a small amount of mode conversion occurs at each interface. In the LL gap, the localized mode consists of a L component with imaginary wave number, and there is a dispersion curve due to the $\mathrm{T}$ component. Furthermore, we can also see the resonance in the TT gap. The T phonons converted from the 
(a)

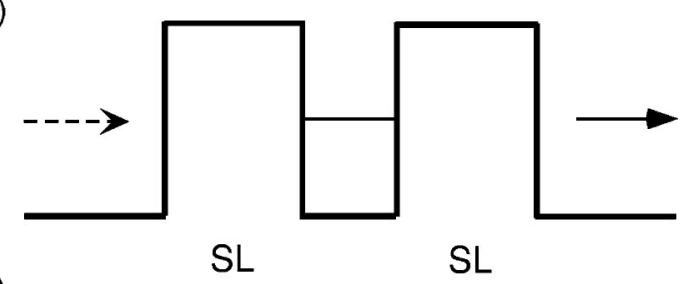

(b)

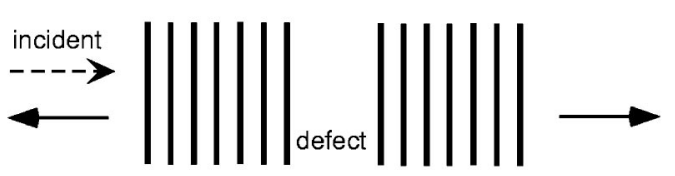

(c)

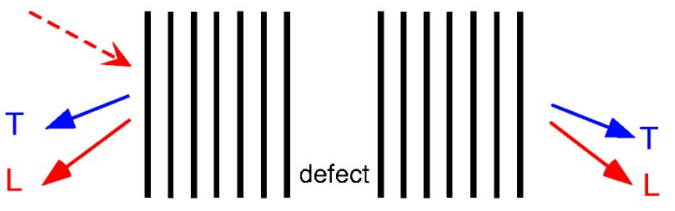

(d)

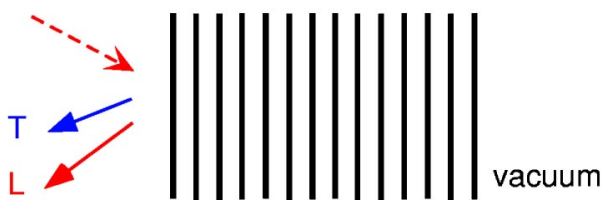

FIG. 3. Schematic diagram of the double-barrier structure and the superlattices with inhomogeneity.

incident $\mathrm{L}$ phonons at each interface resonate with the localized mode consisting of $\mathrm{T}$ components.

The resonant transmission of phonons in the present system can be regarded as a resonant tunneling through a double-barrier quantum-well structure [see Figs. 3(a) and $3(b)]$ because the wave vectors of the phonons within the frequency gaps have an imaginary part in the region of the SL. For electrons tunneling through the double-barrier quantum-well structure, it is well known that the perfect resonant tunneling (with unity transmittance) occurs if the system is symmetric [see Fig. 3(a) ]. ${ }^{15}$ For phonons, this perfect resonant transmission can also occur if the phonons are propagating normal to the interfaces. ${ }^{9}$ For normal incidence, all three phonon modes are decoupled from each other (if the interfaces are a mirror symmetry plane). In this case, incident phonons have only one channel, that is, they are reflected or transmitted with the same polarization [see Fig. 3(b)]. For oblique incidence [Fig. 3(c)], on the other hand, they have two channels (or three channels for the anisotropic media) and perfect resonant transmission does not occur, as shown in Fig. 2.

As a second example, we consider a SL with a free surface. In this system, the phonons injected from a substrate are all reflected irrespective of the frequency though the characteristics of the phonon propagation is frequency dependent. The L phonons injected from the substrate become reflected $\mathrm{L}$ phonons or reflected $\mathrm{T}$ phonons [see Fig. 3(d)]. In other words, they effectively have only one channel. We are particularly interested in the resonant interaction of incident incident phonon : L (a) AlAs surface
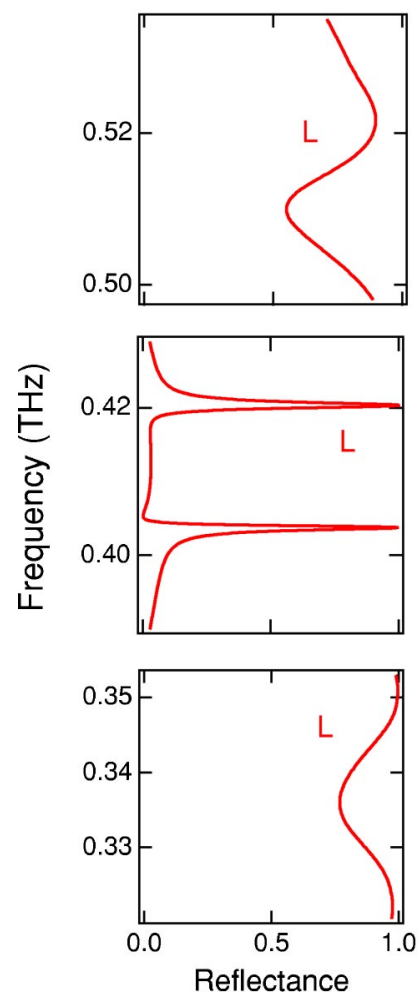

(b) GaAs surface
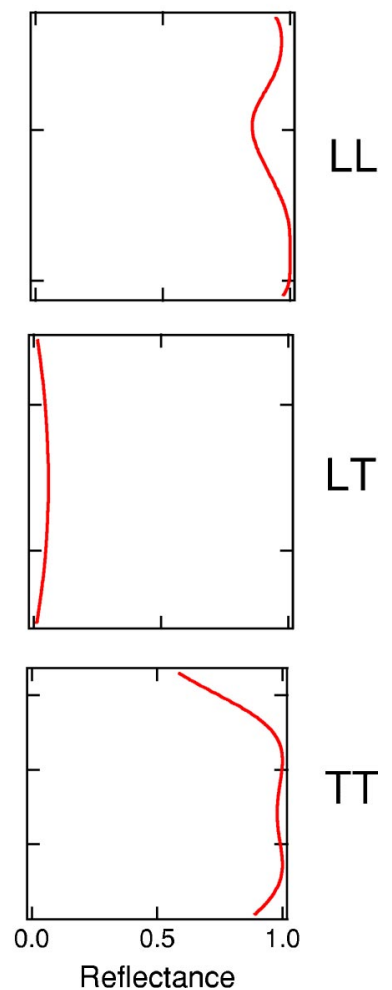

FIG. 4. The reflectance of phonons obliquely incident on a (001) GaAs/AlAs SL with a free surface. The surface layers are (a) AlAs and (b) GaAs. The incident phonons are assumed to be $\mathrm{L}$ phonons. The solid lines are for the reflected L phonons.

phonons with the surface-localized modes generated in the present system. It should be stressed that the present system is considered to be symmetric because the phonons have to make a round trip in this situation. Thus, perfect resonant reflection can be expected. Mode conversion occurs at the surface in addition to the interfaces between constituent layers. Thus, the overall profile of the reflectance spectrum becomes complicated. However, the features inside the gaps are understandable. The calculated reflectance within each frequency gap is shown in Fig. 4. For a SL with a free surface, it is known that surface-localized vibration is only possible when the acoustic impedance of the surface layer is smaller than that of the other layer. ${ }^{5}$ This condition is satisfied for a GaAs/AlAs SL with an AlAs surface layer [Fig. 4(a)]. For comparison, the result calculated for a GaAs/AlAs SL with a GaAs surface layer is shown in Fig. 4(b).

For the SL with a GaAs surface, the incident L phonons with the frequency inside the LL gap are Bragg reflected as $\mathrm{L}$ phonons, that is, intramode Bragg reflections occur, as in the SL without a surface [Fig. 1(b)]. The reflectance of the $\mathrm{L}$ phonons is close to unity. Within the TT gap, L phonons can propagate up to the surface but they are reflected by the surface and come back to the substrate. Within the LT gap, the reflectance of the $\mathrm{L}$ phonons becomes very small since intermode Bragg reflections occur. 
For the SL with a AlAs surface, we can see two resonant peaks within the LT gap. The magnitudes of these peaks are shown to be unity within the numerical accuracy. That is, perfect resonant reflection is realized within the LT gap, as expected. In this frequency window, incident $\mathrm{L}$ phonons are generally reflected as $\mathrm{T}$ phonons. At the eigenfrequencies of the surface-localized mode, however, the additive interference between the incident $\mathrm{L}$ phonons and reflected $\mathrm{T}$ phonons is violated. As a result of resonance, the incident $\mathrm{L}$ phonons are reflected as $\mathrm{L}$ phonons with unity reflectance. This resonance can be interpreted as caused by resonant tunneling through the symmetric double-barrier potential with one channel, as discussed above. Since the LT gap is a real gap, the wave numbers of both $\mathrm{L}$ and $\mathrm{T}$ phonons become imaginary within this gap. On the other hand, the LL (TT) gap is not the real gap, that is, the wave numbers of the L (T) phonons are imaginary but those of $\mathrm{T}(\mathrm{L})$ phonons are real. The $\mathrm{T}(\mathrm{L})$-polarized vibrational mode is extended over the superlattice. Thus, mode conversion at interfaces or at the surface leads to mixing of the localized phonons and extended phonons. As a result, the resonant peak broadens and its magnitude becomes smaller than unity. Perfect resonant reflection occurs only inside the LT gap.

In conclusion, we have examined the resonant interaction of localized modes with acoustic phonons injected into SL's with inhomogeneity at an angle. We demonstrated the difference among the localized modes generated in the LL, TT, and LT gaps. In particular, we found a perfect resonant reflection for phonons scattered by the SL with a free surface. This resonant reflection occurs inside the LT gap. In the LT gap, incident $\mathrm{L}$ phonons are generally reflected as $\mathrm{T}$ phonons because of the intermode Bragg reflection, i.e., the additive interference between the incident $\mathrm{L}$ phonons and reflected $\mathrm{T}$ phonons. This additive interference is broken at the eigenfrequencies of the localized modes. The resonant interaction of the incident phonons with the localized modes generates sharp peaks in reflectance. For the phonons reflected by the SL with a free surface, perfect reflection without mode conversion is realized if the frequencies coincide with the eigenfrequencies of the surface-localized modes. This perfect reflection of phonons can be interpreted as the resonant tunneling through the symmetric double-barrier potential in which the perfect resonant transmission occurs.

It should be noted that the behaviors demonstrated in the present paper are quite general. As a numerical example, we chose a particular incident angle $\left(\theta_{L}=25\right)$. The results given in the LT gaps of Figs. 1, 2, and 4 are essentially independent of the incident angle. In particular, we emphasize that the perfect resonant reflection in the LT gap shown in Fig. 4 occurs also for $\theta_{L} \neq 25$. On the other hand, there is no reason for the perfect resonant reflection to be expected in the LL and TT gaps. In addition, incident phonons are assumed to be $\mathrm{L}$ phonons in the present study. In the case where the incident phonons are $\mathrm{T}$ phonons, the essential results do not change. The results for the incident $\mathrm{T}$ phonons can be explained by exchanging symbols $\mathrm{L}$ and $\mathrm{T}$ in the results for the incident $\mathrm{L}$ phonons. For example, the curves illustrated in Fig. 4 can be interpreted as the results for the reflected $\mathrm{T}$ phonons. Finally, we remark that in anisotropic SL the mode conversion of phonons is more complicated. Mode conversion between two $\mathrm{T}$ modes becomes possible in addition to the mode conversion from the $\mathrm{L}$ to both $\mathrm{T}$ modes. However, as far as we consider the phonon propagation in a sagittal plane of the crystalline structure with mirror symmetry, mode conversion between $\mathrm{L}$ and single $\mathrm{T}$ modes as studied in the present paper is a valid assumption.

The author acknowledges S. Tamura and O. B. Wright for reading the manuscript and giving valuable advice. This work was partly supported by a Grant-in-Aid for Scientific Research from the Ministry of Education, Culture, Sports, Science and Technology of Japan (Grant No. 13650001).
${ }^{1}$ For a compact review, see S. Tamura, in Proceedings of the $3 \mathrm{rd}$ International Conference on Phonon Physics and the 6th International Conference on Phonon Scattering in Condensed Matter, Heidelberg, 1989, edited by S. Hunklinger, W. Ludwig, and G. Weiss (World Scientific, Singapore, 1990), p. 703.

${ }^{2}$ S. Mizuno, Recent Research Developments in Physics (Transworld Research Network, Kerala, 2002), Vol. 3, p. 379.

${ }^{3}$ J. D. Joannopoulos, R. D. Meade, and J. N. Winn, Photonic Crystals (Princeton University, Princeton, 1995).

${ }^{4}$ S. Tamura, D.C. Hurley, and J.P. Wolfe, Phys. Rev. B 38, 1427 (1988).

${ }^{5}$ R.E. Camley, B. Djafari-Rouhani, L. Dobrzynski, and A.A. Maradudin, Phys. Rev. B 27, 7318 (1983).

${ }^{6}$ H.T. Grahn, H.J. Maris, J. Tauc, and B. Abeles, Phys. Rev. B 38, 6066 (1988).
${ }^{7}$ H.J. Trodahl, P.V. Santos, G.V.M. Williams, and A. Bittar, Phys. Rev. B 40, 8577 (1989).

${ }^{8}$ S.I. Tamura, Phys. Rev. B 39, 1261 (1989).

${ }^{9}$ S. Mizuno and S.I. Tamura, Phys. Rev. B 45, 13423 (1992).

${ }^{10}$ M. Giehler, T. Ruf, M. Cardona, and K.H. Ploog, Physica B 263264, 489 (1999).

${ }^{11}$ S. Mizuno, Phys. Rev. B 63, 035301 (2001).

${ }^{12}$ E.H. El Boudouti, B. Djafari-Rouhani, and A. Nougaoui, Phys. Rev. B 51, 13801 (1995).

${ }^{13}$ D. Bria, E.H. El Boudouti, A. Nougaoui, B. Djafari-Rouhani, and V.R. Velasco, Phys. Rev. B 60, 2505 (1999).

${ }^{14}$ D. Bria, E.H. El Boudouti, A. Nougaoui, B. Djafari-Rouhani, and V.R. Velasco, Phys. Rev. B 61, 15858 (2000).

${ }^{15}$ M.Y. Azbel, Phys. Rev. B 28, 4106 (1983). 